\title{
LIV. On flutings in a sound-wave and the forces due to a flux of a viscous fluid around spheres
}

\section{S.R. Cook M.S. A.M.}

To cite this article: S.R. Cook M.S. A.M. (1902) LIV. On flutings in a sound-wave and the forces due to a flux of a viscous fluid around spheres , Philosophical Magazine Series 6, 3:17, 471-482, DOI: $10.1080 / 14786440209462791$

To link to this article: http://dx.doi.org/10.1080/14786440209462791

曲 Published online: 15 Apr 2009.

Submit your article to this journal $[\pi$

Џ Article views: 2

Q View related articles $\square$

Citing articles: 4 View citing articles 5 
Before closing it should be stated that the present research has been greatly facilitated by funds awarded by the Government Grant Committee of the Royal Society. This opportanity is taken to thank Professor Schuster for so kindly placing the ample resources of his laboratory at my disposal; my thanks are also due to the Committee of the Davy-Faraday Research Laboratory for the loan of certain parts of the apparatus. Lastly, it is only just to state that the satisfactory results obtained with the apparatus described are attributed to the care and skilled workmanship of Mr. Charles W. Cook.

LIV. On Flutings in a Sound-Wave and the Forces due to a Flux of a Viscous Fluid around Spheres. By S. R. Cook, M.S., A.M., Former Fellow in Physics, Uninersity of Nebraska; Professor of Physics in Washburn College, Kansas*.

$\triangle$ COUSTIC attractions and repulsions have been carefully 1 studied and much of the accompanying phenomena explained by Messrs. Guyot $\dagger$, Schellbach $\ddagger$, Guthrie $\S$, and Lord Kelvin \|. Attractions and repulsions with the soundwave have been more recently discussed by Walter Koenig $\pi$.

In his discussion Koenig makes use of the hydrodynamical equations for a perfect fluid, and from them deduces formulæ which represent forces brought into action by the flux of a fluid around spheres. He finds that the flux of a perfect fluid around spheres produces a force of repulsion parallel, and attraction perpendicular to the stream-lines; also a movement of rotation tending to move an ellipsoid or disk into a plane transverse to the stream-lines. These forces are considered by Koenig sufficient to explain the formation of laminæ and flutings in the sound-wave.

Although Koenig's explanation has been generally accepted ${ }^{*}$, yet it seemed to the writer that frictional forces must be present, and that opposite forces were necessary to maintain the particles forming the laminæ in their respective positions.

The purpose of this paper was to make a carefnl study

* Piead before the Denver Meeting of the American Association for the Idvancement of Science. Communicated by Prof. D. B. Brace.

+ Presse Srientifique, 1861, t. iii. p. 130 .

I Pogg. Anr. vol. cxxxix. st. 4, p. 670.

ई Phil. Mag. [4] xli. 1871, p. 405.

II Phil. Mag. [4] xli. 1871, p. 423.

Wied. Ann. t. xlii, pp. 358, 549; t. xliii. p. 43 (1891).

* Lord Rayleigl, Theory of Sound, vol. ii. p.46. 
of the formation of laminæ by different fluting materials, with different media under varying conditions, for the purpose of making manifest other forces than those of a perfect fluid, and also to experimentally verify the presence of the forces theoretically deduced from hydrodynamical equations for a perfect fluid.

The apparatus were modified forms of the Kundt soundapparatus. The vibrating brass bar was $277.7 \mathrm{~cm}$. in length, with a frequency of 1287.5 per second and an amplitude of vibration, with ordinary bowing, of $0.2 \mathrm{~mm}$.; the bar being clamped for its first overtone.

In the stationary sound-wave, as that of the organ-pipe or Kundt tube, are to be found two distinct phenomena :-1st, The separation of the tube into nodes and antinodes; 2 nd, the formation in the antinodes or ventral segments of flutings and laminæ. Two theories have been advanced to explain the formation of these laminæ:--1st, They are the nodes of very high overtones produced in the vibrating system; 2nd, they are produced by forces originated by the flux of the medium.

\section{As Nodes of High Overtones.}

The rapid motion of the particles in the laminæ, the movements of these laminx, the breaking up of old and the forming of new laminæ, make it highly improbable that they are produced by overtones. However, in order that this might be more firmly established, a tone was produced by a siren in front of a tube closed at one end by a moving piston. With the proper adjustment of the vibrating air-column, laminæ of magnesium carbonate could easily be produced.

\section{Lamina due to Hydrodynamical Forces.}

Koenig's solution of the hydrodynamical equations for a perfect fluid seem to give a more rational explanation of the problem. The formula obtained by Koenig for the forces between two spheres, whose radii are $R$ and $R_{1}$ and whose distance apart is $r_{0}$, were

$$
\begin{aligned}
& \mathrm{X}=-\frac{\frac{3}{2} \pi \rho \mathrm{R}^{3} \mathrm{R}_{1}{ }^{3} \mathrm{~W}_{0}{ }^{2}}{r_{0}{ }^{4}} \sin \theta\left(1-5 \cos ^{2} \theta\right), . \\
& \mathrm{Z}=-\frac{\frac{3}{2} \pi \rho \mathrm{R}^{3} \mathrm{R}_{1}{ }^{3} W_{0}{ }^{2}}{r_{0}{ }^{4}} \cos \theta\left(3-5 \cos ^{2} \theta\right) ; . .
\end{aligned}
$$

$W_{0}$ being the velocity of the stream, $\rho$ the density of the medium, and $\theta$ the angle which the line of centres of the spheres makes with the direction of the stream. If the 
particle is in the form of an ellipsoid or disk, Koenig finds a moment $M$ tending to diminish the angle $\theta$,

$$
\mathrm{M}={ }_{3}^{4} \rho \mathrm{R}_{2}^{3} \mathrm{~W}_{0}{ }^{2} \sin 2 \theta, \text {. . . . . }
$$

where $R_{2}$ is the radius of the disk.

When $\theta=\frac{n \pi}{2}, n$ being an integer,

When $\theta=n \pi$

$$
\mathrm{X}=-\frac{3}{2} \frac{\pi \rho \mathrm{R}^{3} \mathrm{R}_{1}^{3} \mathrm{~W}_{0}^{2}}{r_{0}^{4}} \ldots . . . .
$$

$$
Z=\frac{3 \pi \rho \mathrm{R}^{3} \mathrm{R}_{1}^{3} \mathrm{~W}_{0}{ }^{2}}{r_{0}{ }^{4}} \text {. . . . . }
$$

This gives repulsion parallel and attraction perpendicular to the stream-lines.

In the equations, the factors susceptible to variation are the density of the medium $\rho$, the size and shape of the particles used for fluting material, $R, R_{1}$, and $R_{2}$, and the stream velocity $W_{0}$.

I shall first allow $\rho$ and $W_{0}$ to remain constant and vary the fluting material.

1. A study of various fluting materials with air as a medium. - The ordinary form of the Kundt sound-apparatus was used; the medium being maintained at normal pressure. Flutings were obtained with the following materials:magnesium carbonate, lycopodium, amorphous silica, cork dust, magnesium, anthracene, ammonium chloride (as fumes), sand, aluminium, brass and iron filings, timothy blue grass, red top, and petunia seed.

After a careful microscopical examination of these materials, it was noticed that as the radii of the particles or disks increased, the mass of the particles remaining the same, the more readily flutings formed; that the more nearly spherical the particles were the more easily they swept into the nodes; and that the greater the radii of the particles the farther apart the laminæ.

2. A study of various fluting material with carbon dioxide and chlorine as media.-With carbon dioxide as medium flutings were obtained in all the substances used with air, and in addition flutings were obtained in filings of coin silver. It was noted that flutings were obtained more easily in carbon dioxide than in air. This was clearly shown by the fact that flutings with coin silver could not be obtained in air. With chlorine as medium, in addition to the above-mentioned substances, flutings were obtained with platinum filings. It is to be noted that in the last two experiments the density $\rho$ 
has been increased, and the forces $\mathrm{X}$ and $\mathrm{Z}$ and the moment $M$ have also been proportionally increased.

We may, however, use the same fluting material so that $R, R_{1}, R_{2}$ will remain constant, and vary the density $\rho$.

3. The ratio of the density of air to the density of carbon dioxide and hydrogen for minimum disturbance. - In order to determine this ratio, it is quite essential that not only the fluting material be exactly the same for the two gases, but also that the stream-velocity $W_{0}$ be the same. This was accomplished by constructing a double-tube Kundt apparatus, one tube at each end of the vibrating brass bar, arranged with manometers and drying trains, and so placed that the tubes were parallel and close to each other. One of these was filled with air ; the other with the gas to be compared. The pressure was then adjusted for minimum disturbance, $i . e$. the pressure at which the fluting material could just be made to form strix. 'The manometers were then read and the observation repeated with gases intershanged.

The results obtained for different fluting material with air and carbon dioxide are given in the following table :-

TABLE I.

\begin{tabular}{|c|c|c|c|c|c|c|c|}
\hline Substance. & T. & N. & $P_{1}$. & $P_{2}$ & $\rho_{1}$ & $\rho_{2}$ & $\rho_{2} / \rho_{1}$ \\
\hline & $\begin{array}{l}20 \\
21 \\
18\end{array}$ & $\begin{array}{r}9 \\
20\end{array}$ & & & & & $\cdot 42$ \\
\hline $\begin{array}{r}\text { Amorph } \\
\text { silies }\end{array}$ & $\begin{array}{l}19 \\
18\end{array}$ & $\begin{array}{r}8 \\
11\end{array}$ & $\begin{array}{l}336 \cdot 8 \\
340.5\end{array}$ & $\begin{array}{l}137 \cdot 5 \\
14222\end{array}$ & & & 62 \\
\hline $\begin{array}{l}\text { Alurninium } \\
\text { filings. }\end{array}$ & $\begin{array}{l}20 \\
22\end{array}$ & $\begin{array}{l}10 \\
10\end{array}$ & $\begin{array}{l}400 \cdot 2 \\
335.0\end{array}$ & $\begin{array}{l}180.5 \\
140.0\end{array}$ & & $\begin{array}{l}.000 \\
.000\end{array}$ & 68 \\
\hline Iycopodium & 21 & 10 & $223 \cdot 3$ & 114.7 & .0008552 & .0002 & $\cdot 77$ \\
\hline Anthracene & 17 & 10 & 21399 & 117.5 & & .000 & 83 \\
\hline San & $\begin{array}{l}20 \\
21\end{array}$ & $\begin{array}{l}10 \\
10\end{array}$ & $\begin{array}{l}641 \cdot 0 \\
627 \cdot 3\end{array}$ & $\begin{array}{l}343 \cdot 5 \\
337 \cdot 3\end{array}$ & & $\begin{array}{l}.0008360 \\
.0008153\end{array}$ & $\begin{array}{r}82 \\
82\end{array}$ \\
\hline
\end{tabular}

$T$ equals the temperature; $N$, the number of determinations in each experiment; $\mathbf{P}_{2}, \mathbf{P}_{2}, \rho_{1}, \boldsymbol{\rho}_{2}$, equal the respective pressures and densities of air and carbon dioxide for minimum disturbance.

It will be seen by the last column that the ratio of the density of carbon dioxide to air is always less than unity. The determination of the ratio of air to hydrogen required great precaution as the slighiest admixture of air would vitiate the results. With the apparatus used the minimum disturbance was obtained at a pressure for hydrogen of $1694 \mathrm{~mm}$. of mercury, and for air at a pressure of $20 \mathrm{~mm}$. of mercury. The corresponding densities were 0001868 and 
0000318 , the ratio of the density of hydrogen to air being $5 \cdot 85$, that of air to hydrogen $\cdot 17$.

In these experiments, since $R, R_{1}, R_{2}, r_{0}$, and $W_{0}$ have been made constant for the two gases, if $X_{1}, Z_{1}$, and $M_{1}$ are the forces of minimum disturbance in air; $\mathrm{X}_{2}, \mathrm{Z}_{2}, \mathrm{M}_{2}$ for the carbon dioxide, then

$$
\frac{X_{1}}{\bar{X}_{2}}=\frac{Z_{1}}{Z_{2}}=\frac{M_{1}}{M_{2}}=\frac{\rho_{1}}{\rho_{2}} ; . . . . .
$$

where $\rho_{1}$ and $\rho_{2}$ are the densities of air and carbon dioxide respectively. And since at minimum disturbance $X_{1}, Z_{1}, M_{1}$ must be equal respectively to $X_{2}, Z_{2}, M_{2}$, then, if there are no forces other than the forces of a perfect fluid, the ratio $\rho_{1} / \rho_{2}$ should be unity ; and since this is not the case, the supposition is that there are other forces which do not vary directly as the density, and since the internal friction of fluids, witbin the limits of these experiments, is independent of the density, it is not improbable that these forces partake of the nature of frictional forces.

For any gas the coefficient of internal friction $\mathrm{R}=k \rho c \lambda$ * and for any other gas of equal density $R_{1}=k \rho c_{1} \lambda_{1}$.

Taking the ratios

$$
\frac{\mathrm{R}}{\mathrm{R}_{1}}=\frac{c \lambda}{c_{1} \lambda_{1}} . \quad \cdot \quad \cdot . \cdot . \cdot
$$

Hence, if there are forces other than the perfect fluid forces proportional to the coefficient of internal friction, they must. also be proportional to the product of the velocity and the mean free path. This led to the study of the intluence of the density on flutings and the ratio of the mean free path to the distance apart of the laminæe.

4. Ratio of the mean free path of the molecule to the distance apart of the lamince.-A Kundt tube was so arranged that the pressure could be reduced to any desired amount and accurately determined. The following table obtains for amorphous silica and air.

\begin{tabular}{|c|c|c|c|c|}
\hline Density. & $\begin{array}{l}\text { Molecular } \\
\text { Velocity C. }\end{array}$ & $\begin{array}{c}\text { Mean free } \\
\text { patb }=\lambda .\end{array}$ & $\begin{array}{l}\text { Distance apart } \\
\text { of flutings }=d \text {. }\end{array}$ & $d / \lambda$. \\
\hline .000180 & 502 & $6114 \times 10^{-8}$ & 72 & 118000 \\
\hline .000228 & 506 & $4748 \times 10^{-8}$ & .50 & 105000 \\
\hline .000360 & 504 & $3077 \times 10^{-8}$ & $\cdot 32$ & 104000 \\
\hline .000695 & 502 & $1557 \times 10^{-8}$ & $\cdot 23$ & 146000 \\
\hline .001152 & 501 & $940 \times 10^{-8}$ & $\cdot 15$ & 159000 \\
\hline
\end{tabular}

TABLE II.

* Boltzmann, Gas Theorie, t. i. p. 81. 
The last column of this table indicates that for a given medium and fluting material there may exist a direct ratio between the distance apart of the flutings and the mean free path of the molecule.

Brief studies on flutings are here presented on account of their peculiar interest in acoustic attractions and repulsions.

5. Flutings produced by a direct sound-wave.-A plane glass plate was substituted for the Kundt tube. The vibrating cork was cut by a horizontal plane and the plate was placed about one millimetre below. When the bar was clamped for its fourth overtone flutings of amorphous silica were produced on the plate. These flutings were elliptical in form, having for their common axis the line between the vibrating cork and the glass plate (fig. 1). Flutings were also produced

Fig. 1.

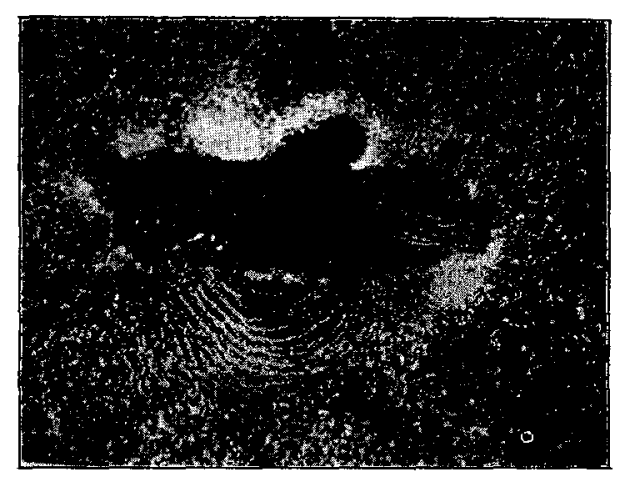

when the bar was clamped perpendicularly and the glass plate placed within a centimetre of the cork. These flutings were concentric circles around the cork (fig. 2).

6. The vibration of lamince.-A mica disk was threaded on a fine wire stretched through the centre of the Kundt tabe. A cork was fastened to one prong of a large tuning-forkfrequency 32 per second-and placed in the end of the Kundt tube. The cork was thus made to vibrate in unison with the prong of the tuning-fork. The mica disk, though in no way connected to the cork, vibrated in unison with it. The amplitude of the vibration of the cork was from one to two centimetres. That of the mica disk was less.

'This experiment suggests that the laminæ might vibrate in unison with the vibrating bar. In order to examine this, a glass tabe was split longitudinally, the edges were ground smooth, and the opening was overlaid with cover-glasses 
sealed around to make them air-tight. A microscope was focussed on the amorphous silica or other substance used for flutings. When the sound-wave was established, the particles of amorphous silica could be seen quickly gathering into thin laminæ which would either sweep out of the field of view or move about in the field with irregular motion.

Fig. 2.

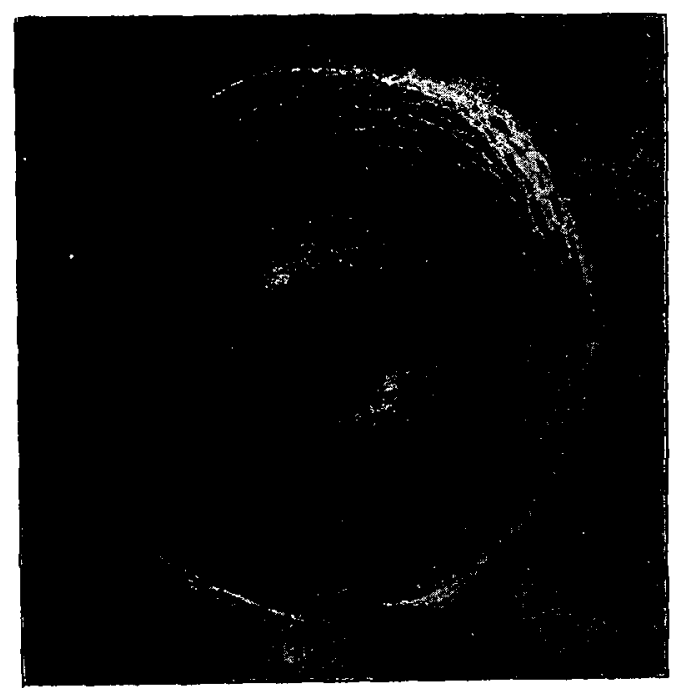

As the number of vibrations of the bar was 1287.5 per second, and the amplitude of vibration for ordinary bowing $0.2 \mathrm{~mm}$., the only means by which a vibration of the laminæ could have been detected would have been by the broadening of the laminæ, and this, when under the microscope, was such a heterogeneous mass of moving particles that no vibration could be detected. The study became very interesting, however, in watching the motion of the individual particles. A larger particle would move towards a smaller particle until within a certain distance, when the small particle would suddenly move away. Again, a particle might be swept up close to another until it seemed to be within another sphere of action, and be drawn to it. The two would then take a position with their line of centres transverse to the streamlines. Particles lying in a transverse position at some distance apurt would be attracted, always assuming the laminal condition. It was noted, however, that these particles, with their spheres of attraction and repulsion, Phil. Mag. S. 6. Vol. 3. No. 17. May 1902. $2 \mathrm{~K}$ 
appeared never to come into actual contact, and it was distinctly evident that they were not in actual contact in the laminæ.

If only the three forces deduced from the equations of a perfect fluid are to be considered, the particles should at least be in contact transversely. After a careful study of these phenomena it was decided, if possible, to obtain spheres small enough and light enough to flute.

\section{Forces due to a Flux of a Viscous Fluid around. Spheres.}

The three forces deduced from the theory of a perfect fluid have already been discussed, and the experiments have pointed to other forces. The presence of these new forces will be more clearly manifest in the following experiments with spheres in a viscous fluid.

The spheres used were obtained by blowing out from a glass tube melted sealing-wax, and allowing the hot spray to filter through the air into a vessel of water. In this manner a large number of variously-sized spheres could be quickly and easily obtained. The spheres, when placed under a microscope, were found to be very perfect in form, with smooth surfaces, and having a diameter of from $0 \cdot 1$ to $2 \cdot 0 \mathrm{~mm}$. When placed in the resonance-tube of the Kundt apparatus, spheres whose diameter was not more than $1 \mathrm{~mm}$. readily formed flutings. A careful microscopic study was then made of attractions and repulsions of these spheres in the centre of the ventral segment of a stationary sound-wave, and the following observations were determined :-

\section{Observations on a Concave Surface.}

(1) Spheres whose line of centres was parallel to the stream-lines and whose distance apart was greater than between one and two tenths of a millimetre repelled.

(2) Spheres whose line of centres was transverse to the stream-lines and whose distance apart was greater than about balf the diameter of the spheres attracted.

(3) Spheres whose line of centres was transverse to the stream-lines and whose surfaces, owing to the concavity of the tube, were in contact when there was no sound-wave present, did not remain in contact when the sound-wave obtained but were driven by some repellant force farther up the side of the tube.

(4) Spheres whose line of centres was parallel with the stream-lines and whose distance was less than half their 
diameter rotated as a single body until their line of centres wis transverse to the stream-lines.

The roughness and shape of the bottom of the tube interfered somewhat with the best observation. To overcome this difficulty a plane piece of plate-glass was fringed with paper, to prevent the spheres from rolling off, and placed in the tube. The spheres resting on the surface of the glass readily formed flutings across its plane surface, but by slightly tilting the glass surface the spheres could be made to roll from side to side where flutings would be formed projecting somewhat up the incline. With this arrangement of the apparatus the following observations were determined:-

\section{Observations on a Plane Surface.}

(1) Observations (1) and (2) of the previous section were verified.

(2) Two spheres with line of centres transverse to streamlines were attracted and then repelled to a distance of about half their diameter, and remained thus while moving forward and back on the glass.

(3) Three spheres situated as in (2) acted in all respects like two spheres.

(4) The surface of the glass plate was inclined at an angle of about $20^{\circ}$ to the horizontal. One sphere was resting against the side of the tube, another was in contact with it, resting on the inclined plate. Their line of centres was transverse to the stream-lines. When the sound-wave was present the second sphere was repelled to a distance of about half its diameter up the incline from the first, and maintained that position during the duration of the sound-wave, then returned to its original position. Three spheres in line acted in a similar manner.

(5) Glass plate as in (4). First sphere fixed to upper side of plane surface. 'The two spheres in relatively the same position as in (4). When the sound-wave ceased the second sphere would start to roll down the inclined surface from the first sphere, but as soon as the sound-wave was again started this sphere was attracted up the inclined plane to within a distance of about half its diameter, where it was maintained during the maintenance of the sound-wave.

Although the sealing-wax spheres seemed to move with perfect freedom on the surface of the plate-glass, and there seemed to be nothing that could possibly vitiate the results obtained, yet the question of possible surface-conditions arose, and it was decided to suspend these same spheres in the centre of the tube. 


\section{Observations with Suspended Spheres.}

A small hole was blown at the centre of a ventral segment in the Kundt resonance-tube, and a small cork was fitted flush with the inner surface of the tube. To this cork two spheres were suspended on the end of fine spider-threads about two centimetres in length, and at any desired distance apart.

Two spheres, whose diameter was about $0.9 \mathrm{~mm}$., thus suspended, with their line of centres parallel to the streamlines, were repelled, but with their line of centres perpendicular to the stream-lines they were attracted. When the line of centres was at an angle of about $45^{\circ}$, or less, they would rotate their line of centres perpendicular to the streamlines and approach each other.

The sealing-wax spheres were replaced by glass spheres of the same size. The results were the same, bat not so pronounced.

These observations were repeated with other sealing-wax spheres, whose diameters were from $\cdot 5 \mathrm{~mm}$. to $\cdot 7 \mathrm{~mm}$., their distance apart being from $1 \mathrm{~mm}$. to $8 \mathrm{~mm}$. The same results were observed.

Spheres of from $\cdot 2 \mathrm{~mm}$. to $\cdot 3 \mathrm{~mm}$. were then suspended in like manner with similar results, except that the forces were more pronounced. With these small spheres suspended within half of their diameter apart, it was observed that the suspensions approached each other when the spheres were in contact.

In order to eliminate this attraction of the fibres, one of the spheres was mounted on the end of a fine glass fibre, which was brought in from the side of the Kundt resonancetube, and the cther was mounted on a single fibre-suspension as before.

When the two spheres were brought within a distance of about half their own diameter, with their lines of centres perpendicular to the stream-lines, the suspended sphere was repelled from the sphere mounted on the glass fibre. When their line of centres was parallel to the stream-lines, and their distance apart less than half their diameter, the suspended sphere approached the fixed sphere and moved around until their line of centres was perpendicular to the streamlines.

These observations were verified by other sets of spheres in other ventral segments.

\section{Influence of Surface on the Forces between two Spheres.}

In order to determine whether or not a surface would 
influence the forces between two spheres, a cover-glass was mounted on the end of a glass rod, and was made to approach the spheres from below. It was found that when the coverglass was very close to the spheres, the attraction parallel and repulsion transverse to the stream-lines was increased.

\section{Discussion of the Data.}

The data here presented have experimentally verified the presence of the forces deduced from the theory of a perfect fuid, and make manifest other forces. The presence of these new forces, which are opposite in direction to the perfect fluid forces, were indicated in the experiments with different gases, but in the observations with spheres the effect of these forces has not only been seen, but the relative magnitude of the two forces has been roughly measured, so that the function of the distance apart of the spheres varies more rapidly than the perfect fluid function.

The experiments with different gases, whose coefficient of viscosity varies, the increase of these forces on the approach of a plane, and the fact that they are opposite in direction to the perfect fluid forces, indicate that these forces are due to internal friction or viscosity of the fluid used as medium.

\section{Formation of Lamina.}

As has already been stated, Koenig considered the two forces, and the moment deduced from the theory of a perfect fluid, sufficient to explain the formation of the laminæ produced in the ventral segment of a Kundt's resonance-tube. If no other than the attractive force existed between particles whose line of centres is perpendicular to the stream-lines, the particles would, in the transverse plane, only be in stable equilibrinm when they are in actual contact. But this condition is not in accord with the most careful observation both of particles and spheres. If, however, there is a repulsive force which is only effective when the particles are not more than one half their diameter apart, there will be a position in the transverse plane in which two particles are in stable equilibrium, and this position is the position in which the particles will be found in the laminæ.

And if in the direction parallel to the stream-lines we consider only a repulsive force, all particles having their lines of centres parallel to the stream-lines would be repelled from each other regardless of their distance apart ; but this does not agree with observation for particles or spheres lying within about $\cdot 3 \mathrm{~mm}$. apart. Particles thus situated act as $a$ 
single system, and are rotated by the moment $M$ into the plane perpendicular to the stream-lines.

The attractive force parallel to the stream-lines, in conjunetion with the moment of rotation, causes the particles which are within the sphere of action to form in laminæ transverse to the stream-lines; and the attraction and repulsion perpendicular to the stream-lines maintain these particles in the laminæ. If, however, there are particles in the plane parallel to the stream-lines, and not within the sphere of action of sufficient intensity, they will be repelled to some greater distance, and may come within the sphere of action of some other particles, and with them form other laminæ.

\section{Summary.}

1.-The flutings are not confined to the ventral segment of a stationary sound-wave, but the conditions for the formation of laminæ are found wherever there is a to and fro flux of a viscous fluid around solid particles.

2. -The ease with which flutings may be formed depends upon the size, shape, and specific gravity of the flutingmaterial, and also upon the molecular weight, density, and viscosity of the medium.

3.-A perfect fluid contains forces which are essential but are not sufficient to produce laminæ in the form in which they exist.

4.-The experiments here noted show that there is another and opposite force both parallel and perpendicular to the stream-lines.

5.-The attractive and repulsive forces are increased by the presence of a surface in close proximity to the solid particles.

6.-These new forces are most probably due to the viscosity of the medium.

7.-The now forces, in conjunction with the forces of the perfect fluid, make possible a complete and clear explanation of laminæ and flutings produced in sound-waves.

My sincere thanks are due to Dr. D. B. Brace for many valuable suggestions and for the interest manifested in the results.

Physical Laboratory, University of Nebraska,

Lincoln, Nebraska, Aug. 22, 1901. 